\title{
LIFESTYLE HABITS AND OBESITY IN A TRANSYLVANIAN TRADITIONALIST ROMA POPULATION VERSUS NON-ROMA POPULATION
}

M. Szabo, T. Komsa, S. Szikszai, T. Kovacs, Z. Tanko, A. Balazs, K. Zsido, B. Máté.

${ }^{1}$ University of Medicine and Pharmacy, Emergency County Clinic Tg Mures, Tg Mures,

Romania

\section{Background.}

- In Transylvania, Romania the prevalence of the Roma is $4.5 \%$.

- Genetic traits and lifestyle of the Roma population differs from that of the Caucasoid population, and their metabolic risk is higher.

- Lifestyle habits among the different Roma subgroups differ a lot,

- The Gabor Roma's are a traditionalist subgroup of rural living Transylvanian Gypsies, neo-protestants, non-smoking, low alcohol consumption, with relatively good financial circumstances.

\section{Method}

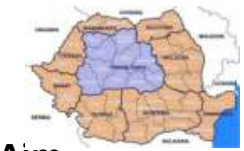

Aim.

To evaluate the metabolic risk parameters (eating habits, physical activity, sleeping habits) and obesity of a traditionalist Roma group, the Gabor's, with less lifestyle related cardiovascular risk factors a compared to neighboring nonRoma's.

A representative sample of Gabor Roma and non-Roma from a rural surrounding in Transylvania were included.

A lifestyle and social life related 70 items questionnaire and anthropometric measurements (body mass index, waist circumference) were performed. Data were collected regarding meal frequency, eating, drinking, physical activity habits and sleeping habits

\begin{tabular}{|l|l|l|l|}
\hline & Gabor roma (331) & $\begin{array}{l}\text { Non-roma } \\
(383)\end{array}$ & \\
\hline Mean age (years SD) & $40.5 \pm 18.03$ & $42.4 \pm 19.3$ & \\
\hline Gender (\% men) & 48.6 & 45.9 & \\
\hline Fr. of meals (nr/day) & 2.6 & 3.2 & .05 \\
\hline Use of water (I/day) & $1.2 \pm 0.7$ & $1.56 \pm 0.6$ & .05 \\
\hline Use of soft drinks & $0.65 \pm 1.3$ & $0.11 \pm 0.2$ & .001 \\
\hline Oil and fat (I/day) & $0.098 \pm 0.013$ & $0.073 \pm 0.007$ & .05 \\
\hline Meat (\% of day use) & 84.4 & 70 & .05 \\
\hline $\begin{array}{l}\text { Fish (\% of weekly } \\
\text { use) }\end{array}$ & 4.3 & 26.3 & .003 \\
\hline Bread (slices/day) & $3.31 \pm 1.7$ & $4.35 \pm 1.8$ & .01 \\
\hline $\begin{array}{l}\text { Fruits (\% of not } \\
\text { eating daily) }\end{array}$ & 33.8 & 5 & .007 \\
\hline $\begin{array}{l}\text { Vegetables (\% of not } \\
\text { eating daily) }\end{array}$ & 34.2 & 0 & .000 \\
\hline $\begin{array}{l}\text { Physical activity } \\
\text { (min/week) }\end{array}$ & $117.32 \pm 96.3$ & $186.84 \pm 146.2$ & .05 \\
\hline $\begin{array}{l}\text { Active in gardening } \\
\text { (\%) }\end{array}$ & 21.1 & 65 & .000 \\
\hline
\end{tabular}

\begin{tabular}{|l|l|l|l|}
\hline & $\begin{array}{l}\text { Gabor roma } \\
(331)\end{array}$ & $\begin{array}{l}\text { Non-roma } \\
(383)\end{array}$ & $p$ \\
\hline $\begin{array}{l}\text { Sleeping } \\
\text { hours } \\
\text { (h/day) }\end{array}$ & $7.39 \pm 1.77$ & $7.38 \pm 1.85$ & $\mathrm{~ns}$ \\
\hline BMI $\left(\mathrm{kg} / \mathrm{m}^{2}\right)$ & $31.53 \pm 4.3$ & $28.54 \pm 5.2$ & .01 \\
\hline WC $(\mathrm{cm})$ & $106.3 \pm 10.2$ & $100.2 \pm 11.1$ & .02 \\
\hline $\begin{array}{l}\text { General } \\
\text { practitioner } \\
\text { (\% never } \\
\text { attending) }\end{array}$ & 32 & 19 & .04 \\
\hline
\end{tabular}

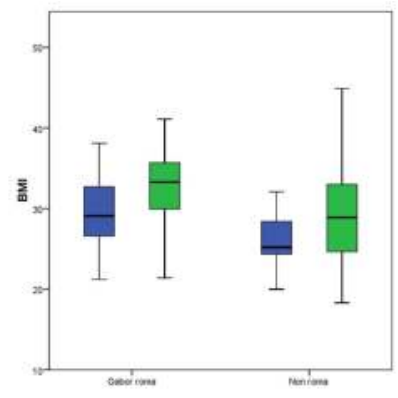

\pm Fig.1.Higher $\mathrm{BMI}$ of women in both ethnic groups
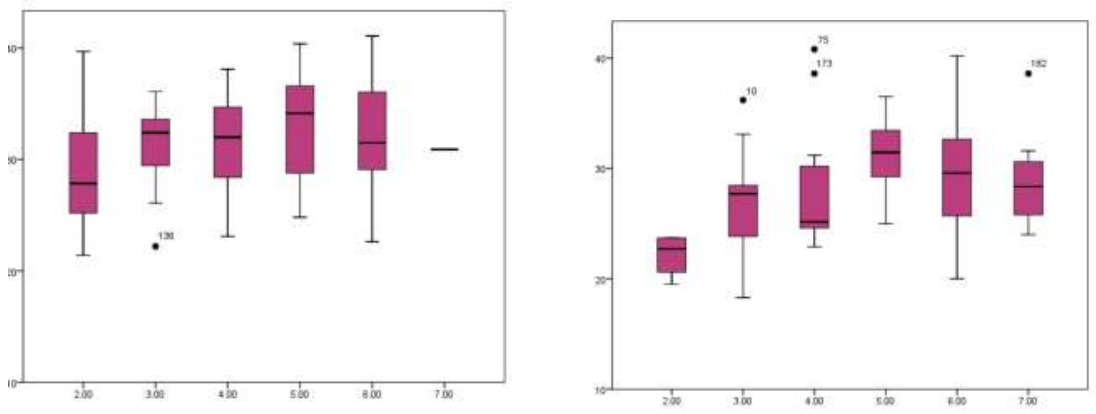

Fig.2. and 3. BMI in different age groups (decades) in Gabor Roma and non-Roma.

Discussion. Although the Gabor's lack certain risk behaviors (smoking, pork meat and alcohol consumption), their eating and physical activity habits differ from those living around them, and are highly unhealthy (lot of soft drinks, oil, less fruit, vegetables, fish). They, and mainly the men, exercise little. They are doing less gardening or sports. They have higher BMI and WC, and obesity is present already at an early age.

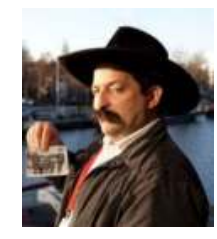

